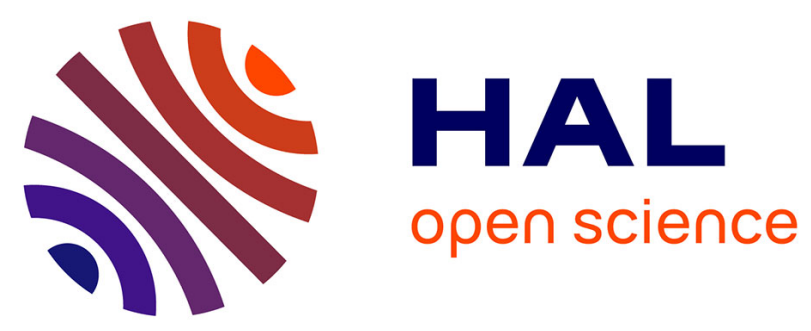

\title{
DRC 2: Dynamically Reconfigurable Computing Circuit based on Memory Architecture
}

Kaya Can Akyel, Henri-Pierre Charles, Julien Mottin, Bastien Giraud, Suraci Grégory, Sebastien Thuries, Jean-Philippe Noel

\section{- To cite this version:}

Kaya Can Akyel, Henri-Pierre Charles, Julien Mottin, Bastien Giraud, Suraci Grégory, et al.. DRC 2 : Dynamically Reconfigurable Computing Circuit based on Memory Architecture. IEEE International Conference on Rebooting Computing, Oct 2016, San Diego, France. cea-01376554

HAL Id: cea-01376554 https://hal-cea.archives-ouvertes.fr/cea-01376554

Submitted on 5 Oct 2016

HAL is a multi-disciplinary open access archive for the deposit and dissemination of scientific research documents, whether they are published or not. The documents may come from teaching and research institutions in France or abroad, or from public or private research centers.
L'archive ouverte pluridisciplinaire HAL, est destinée au dépôt et à la diffusion de documents scientifiques de niveau recherche, publiés ou non, émanant des établissements d'enseignement et de recherche français ou étrangers, des laboratoires publics ou privés. 


\section{DRC $^{2}$ : Dynamically Reconfigurable Computing Circuit based on Memory Architecture}

Kaya Can Akyel, Henri-Pierre Charles, Julien Mottin, Bastien Giraud, Grégory Suraci, Sébastien Thuries and Jean-Philippe Noel CEA, LETI, MINATEC Campus, F-38054 Grenoble, France, Univ. Grenoble Alpes, F-38000 Grenoble, France

kayacan.akyel@cea.fr

\begin{abstract}
This paper presents a novel energy-efficient and Dynamically Reconfigurable Computing Circuit $\left(\mathrm{DRC}^{2}\right)$ concept based on memory architecture for data-intensive (imaging, ...) and secure (cryptography, ...) applications. The proposed computing circuit is based on a 10-Transistor (10T) 3-Port SRAM bitcell array driven by a peripheral circuitry enabling all basic operations that can be traditionally performed by an ALU. As a result, logic and arithmetic operations can be entirely executed within the memory unit leading to a significant reduction in power consumption related to the data transfer between memories and computing units. Moreover, the proposed computing circuit can perform extremely-parallel operations enabling the processing of large volume of data. A test case based on image processing application and using the saturating increment function is analytically modeled to compare conventional and $\mathrm{DRC}^{2}$-based approaches. It is demonstrated that $\mathrm{DRC}^{2}$-based approach provides a reduction of clock cycle number of up to $2 x$. Finally, potential applications and must-be-considered changes at different design levels are discussed.
\end{abstract}

Keywords-in-memory computing, computing architecture, programmable logic

\section{INTRODUCTION}

The growing speed gap between memories and computing units in Von Neumann architecture, also known as the "memory wall", has led to many research on associative memory circuits and alternative computing architectures [1][2]. Register-heavy solutions were one of the approaches developed in order to overcome this bottleneck without any fundamental changes in processor architecture. The well-known Graphical Processing Units (GPU) are a good example of computing circuits using a register-heavy memory hierarchy (few $\mathrm{Mb}$ of register files compared to few $\mathrm{Kb}$ in a mainstream $\mathrm{CPU}$ ) including a very large number of registers files (RF), which allow highly-parallel computing. Moreover, the architecture of SRAM-based RFs itself is also optimized and multi-port bitcells are introduced offering simultaneous read (RD) and write (WR) operations [3][4] to further increase the computing speed. However, parallelization of many computing units and the resulting high throughput of the data leads to a high power consumption [5]. Indeed, with the increase of wire parasitic elements at each new technology node (due to the shrink of the metal routing pitch), the power dissipated by the large amount of data moving between memories and computing units across these highlyparasited wires becomes more and more critical. This resulting high power consumption is seen as one of the principal constraint in contemporary embedded circuit design. As an example, fetching operands in a $28 \mathrm{~nm} n$ Vidia GPUs costs more than computing the operation itself. Thus, this ratio can rise up to $1000 \mathrm{x}$ depending on the location of the data to be fetched in the on-chip memory hierarchy [6][7]. As a result, decreasing data-transfer related power has become a hot research topic. Shortening the physical distance between memories and processing elements thus appears as a relevant solution to reduce the total wire length and thus the overall power consumption.

Processing-in-Memory (PIM) concept [8] has been introduced for this purpose to bring the processing elements into the same die as DRAM, thus addressing primarily to off-chip memories. PIM concept, which limits both the latency between memory a)

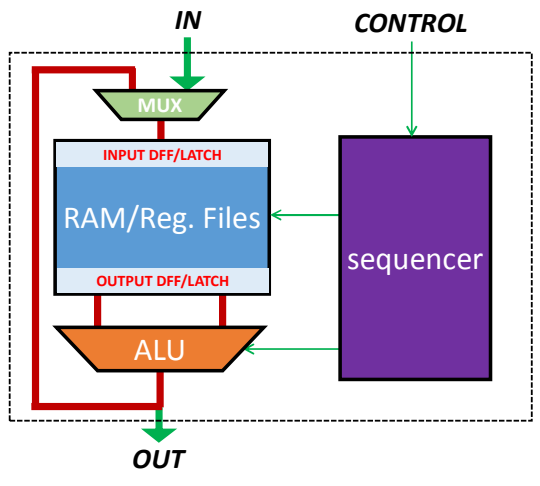

b)

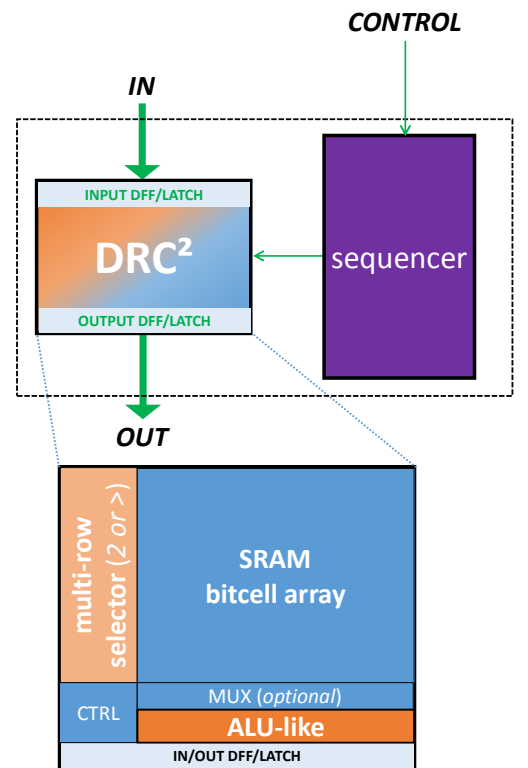

Figure 1 a) Conventional computing architecture vs. b) in-memory computing architecture. Moving into In-memory computing should allow speed increase, total area saving and less power consumption since physical distance between memory and computing unit is drastically reduced. 


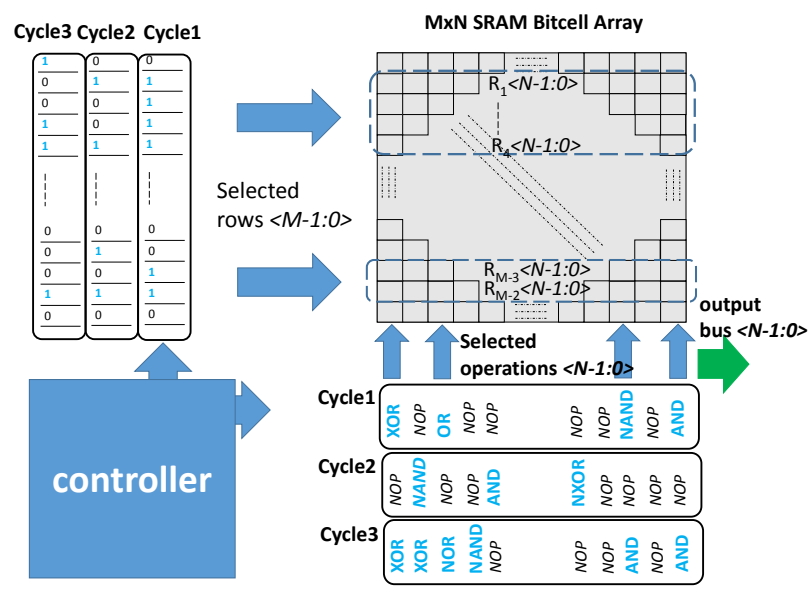

Figure 3 Proposed Dynamically Reconfigurable Computing Circuit (DRC $\left.{ }^{2}\right)$ based on 3-Port SRAM bitcell. Instead of delivering the stored content, the output bus delivers computed metadata.

and processing unit and the resulting dissipated power, has been reborn recently thanks to the 3D stacking [9]. Some works, as in [10], investigated similar approach for on-chip SRAM circuits, which are used as processor caches due to their significantly higher speed (compared to DRAM). With the rise of emerging NVM technologies, memristor-based material implication (IMPLY) function is proposed to perform Computing-inMemory (CiM) [11][12], enabling same physical location for both storage and computing. However, memristor-based IMPLY results in destructive operations meaning that the initially stored data is lost after computing.

Another way to drastically increase the computing speed, while not increasing overall power (and area), is to use alternative nonVon Neumann architectures [13]. A suitable architecture for an energy-efficient computing also requires re-configurability since rising chip development costs make workload specific chips very expensive products [14]. At this end, Intel announced that the latest version of their Xeon server chip will have an integrated FPGA to boost performance under workloads requiring optimized processing. An important point in the literature is that SRAM circuits are discarded for long time and spared from any modification, while solutions are sought in high-level design. This is probably because SRAM bitcell arrays are designed with the most aggressive design rules in a given technology and suffer from stability issues due to the process variability. Thus, any change in the array can lead to a highly complex manufacturability issues. However, the need for more optimizations to significantly increase the computing speed at the same time with re-configurability encouraged redesign of SRAM macros with novel features such as in-memory logic function operations [15].

In this work, the CIM concept is pushed one step further and a novel computing circuit is proposed enabling in-memory logic and arithmetic operations exploiting the architecture of SRAM macro, thus lowering data transfer between memory and computing unit (i.e. ALU), as shown in Figure 1. The proposed Dynamically Reconfigurable Computing Circuit $\left(\mathrm{DRC}^{2}\right)$ can perform the same logic and arithmetic operations as a basic ALU and, therefore enables non-destructive computing and storage in the same unit. Owing to this approach, we expect significant gains in power consumption and thus pave the way for new opportunities in computing, in particular by using the dynamic reconfigurability since operations are computed in-situ, without using "frozen" dedicated computing circuits based on logic gates.

The paper is structured as follows: The section II introduces the concept of proposed DRC ${ }^{2}$. The section III presents the 3-port 10T bitcell used in the SRAM array and how different logic and arithmetic operations can be performed. Then a representative test case based on $\mathrm{DRC}^{2}$ for imaging processing is discussed in section IV. Perspectives for the proposed concept are briefly discussed in section V. Finally, section VI concludes the paper.

\section{Dynamically ReConfigurable Computing Circuit $\left(\mathrm{DRC}^{2}\right)$ CONCEPT}

Figure 3 presents the $\mathrm{DRC}^{2}$ concept. It is based on a SRAM bitcell array in which multiple rows can be selected in the same time during the same clock cycle for performing in-situ operations between selected rows [15]. The operation type is chosen and controlled slice-wise (using a dedicated controller, as shown in Figure 3), thus different operations can be performed on different slices of the same array offering highlyparallel operations. For the rest of the paper, the term "memory slice" is used to describe a memory column with some selected bitcells and the associated column peripheral circuitry. The results coming from a given memory slice can be read as in any conventional memory circuit, or can be written-back (WB) into the memory in the same or next clock cycle.

Table 1 presents the list of 19 basic operations that can be performed by the proposed $\mathrm{DRC}^{2}$. It enables to perform these operations inside the memory, thus limiting intensive data transfert. Operations like RD, RD_NOT, RD_0 and RD_1 are natively present in a conventional memory circuit (or can be added without any complexity), unlike to the others. As shown in Figure 3, the SRAM bitcell array is accessed through multiple rows in the same time in the same clock cycle, which is indeed prohibited in a conventional memory circuit to ensure a proper read. The multiple row access being the key for computing inside the memory circuit, it is worth to say that the intelligence

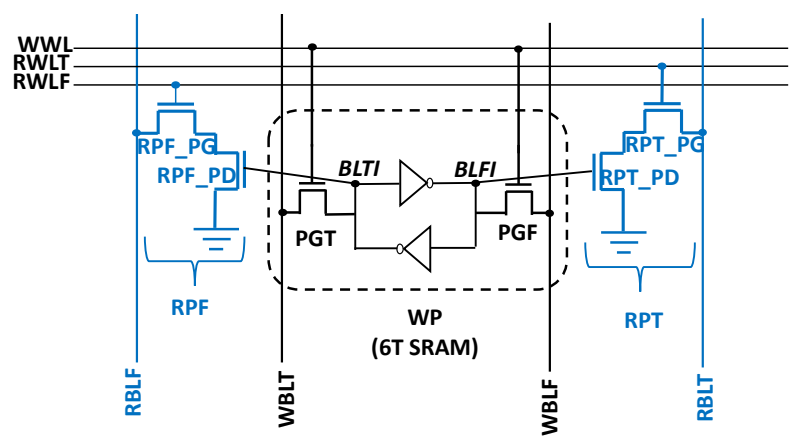

Figure 2 10-Transistors (10T) SRAM bitcell including one Write-Port (WP) and two Read-Port (RP), False (F) and True (T). Each RP is formed by its pass-gate (PG) and its pull-down (PD) transistors. 
TABLE I

\begin{tabular}{|l|l|l|l|l|}
\hline Operation & Description & \# of operands & \# of cycle & Operation Type \\
\hline RD/BUFF & Read the content of selected bitcell & 1 & 1 & Memory \\
\hline $\begin{array}{l}\text { RD_NOT/IN } \\
\text { V }\end{array}$ & RD the complementary content of selected bitcell & 1 & 1 & Memory \\
\hline RD_0 & Read as "0" the selected bitcell & 1 & 1 & Memory \\
\hline RD_1 & Read as "1" the selected bitcell & 1 & 1 & Memory \\
\hline $\begin{array}{l}\text { XOR/ } \\
\text { COMP }\end{array}$ & $\begin{array}{l}\text { Exclusive OR if \#of operands =2 } \\
\text { Comparison if \# of operands >2 }\end{array}$ & N & 1 & Logic \\
\hline NXOR & NOT Exclusive OR & N & 1 & Logic \\
\hline NOR & NOT-OR & N & 1 & Logic \\
\hline NAND & NOT-AND & N & 1 & Logic \\
\hline OR & OR & N & 1 & Logic \\
\hline AND & AND & N & 1 & Logic \\
\hline IMP & Materiel Implication & N & 1 & Logic \\
\hline SHIFT & Shift the bitcell content to left or right & 1 & 2 & Logic/Arithmetic \\
\hline ADD & Addition & 2 & 3 & Arithmetic \\
\hline SUB & Subtraction & 2 & 4 & Arithmetic \\
\hline INC & Increment by 1 & 1 & 3 & Arithmetic \\
\hline DEC & Decrement by 1 & 1 & 3 & Arithmetic \\
\hline GT & Greater than & 2 & 2 & Arithmetic \\
\hline LT & Less than & 2 & 2 & Arithmetic \\
\hline
\end{tabular}

Table 1 List of operations, their description, number of maximum operands, number of minimum cycles that is needed to perform these operations and operation family that they are belonging.

comes from both the bitcell array access and the associated peripheral circuitry.

\section{DESCRIPTION OF OPERATIONS ENABLED BY DRC ${ }^{2}$}

Figure 2 presents the 10-Transistors (10T) SRAM bitcell used in the array. It is composed of one 6T write-port (WP) and two $2 \mathrm{~T}$ read-port (RP). Internal nodes BLTI (stores data) and BLFI (stores complemented data) of WP are connected to RP False (RPF) and RP True (RPT), respectively, allowing isolation of read and write operations. Both RPs are connected to their proper read bit-lines (RBLF and RBLT, respectively), through pass-gate (PG) transistors RPF_PG and RPT_PG. RPF and RPT are connected through pull-down (PD) transistor RPF_PD and RPT_PD to ground line GND.

This 10T SRAM bitcell is accessed through three Word-Line (WL) signals, as shown in Figure 2: Write WL (WWL), Read WL True (RWLT and Read WL False (RWLF). WWL allows accessing internal nodes of 6T WP, thus used for write operation (WR). WR is performed as in any conventional SRAM by activating corresponding WWL and driving WBLs at the values to be written in the bitcell. RWLT allows selecting RPT, thus reading the stored data through BLFI without a risk of an

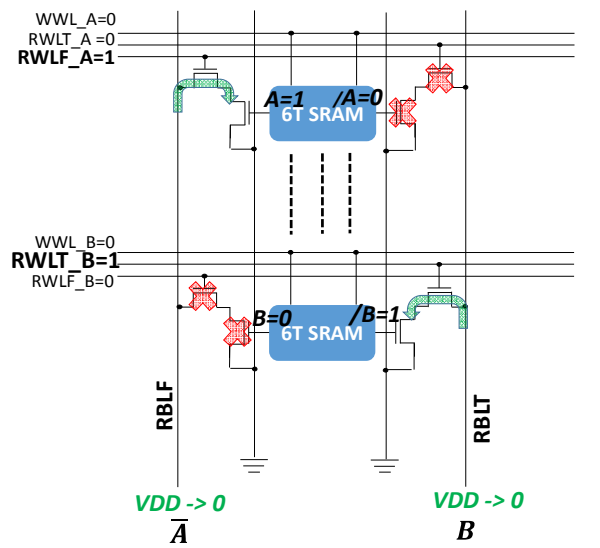

a) $R D(\operatorname{INV}(A))$ and $R D(B)$ in the same cycle conventional $2 R$ mode

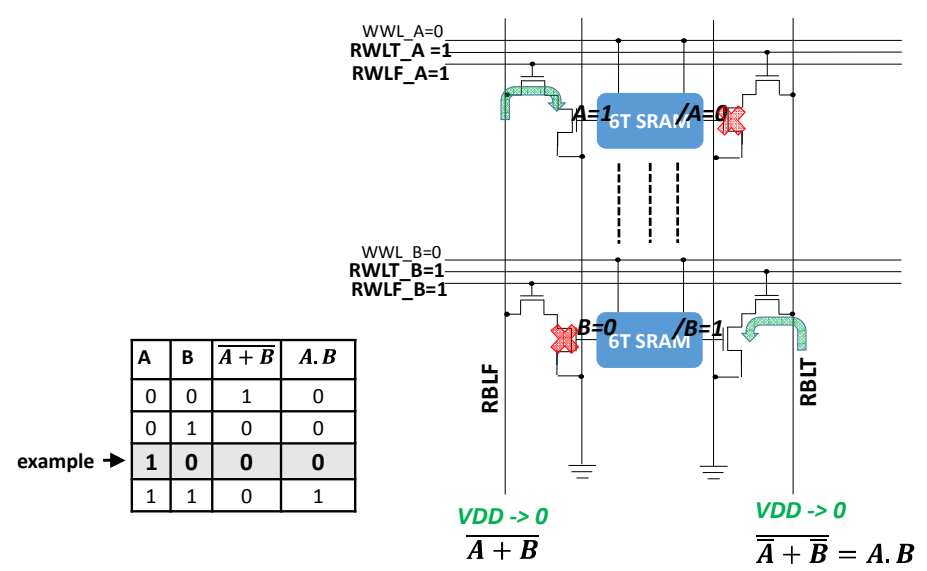

b) $\operatorname{NOR}(A, B)$ and $\operatorname{AND}(A, B)$ in the same cycle Multiple-row access

Figure 4 a) Dual access to memory slice (through RPF and RPT) allowing to perform RD(NOT(A)) and RD(B) on RBLF and RBLT, respectively. b) Multiple access through RPF and RPT allowing to perform NOR $(A, B)$ operation on RBLF and AND(A,B) operation on RBLT. For the sake of simplicity, only NOR and AND operations with two operands are shown. Both operations can be performed with up to $\mathrm{M}$ operands where $\mathrm{M}$ is the column length. 
unwanted data flip inside the WP (read disturb phenomena [17]), while RWLF allows reading the complemented data through BLTI and RPF. The used nomenclature for RPT and RPF comes from following: BLTI stores the true data and BLFI stores the complemented data, i.e. false data. The value read through RPF is data stored in BLFI, while the data read through RPT is data stored in BLTI.

In this work, the 10T SRAM bitcell is chosen since we consider that it is the most efficient bitcell for the proposed computing circuit. However, any other bitcell allowing differential read (single-port 6T, dual-port 8T, etc.) can be used for the same purpose at the expense of not being able to perform write and read in the same clock cycle, which impacts the overall number of cycles depending on the desired operations.

\section{A. RD, NOR/OR and AND/NAND operations}

Figure 4.a illustrates a conventional dual RD operation in $10 \mathrm{~T}$ SRAM memory slice through both RPs: RBLF and RBLF are pre-charged to VDD prior to the operation. The bitcell $\mathrm{A}$ is selected by activating (from 0 to 1 ) RWLF_A and the bitcell $\mathrm{B}$ is selected by activating RWLT_B, while other RWLs are kept low. Which means that all pass-gate transistors are OFF in the relevant memory column except RPF_PG of bitcell A and RPT_PG of bitcell B. In this example, bitcell A stores a ' 1 '

(BLTI=VDD, BLFI=GND), and bitcell B stores a ' 0 ' (BLTI=GND, BLFI=VDD). As a result, RPF_PD of bitcell A and RPT_PD of bitcell B are both ON and lead to the discharge of RBLF and RBLT, respectively through RPs. In other words, the final voltage level of RBLF is equal to $\bar{A}$, while the final voltage level of RBLT is equal to $B$.

Figure 4.b illustrates the same memory slice bit with multiple row access in which only two bitcells, A and B, are shown. Like a conventional read operation, RBLF and RBLT are pre-charged to VDD. Then, bitcells $\mathrm{A}$ and $\mathrm{B}$ are accessed through their respective RPF and RPT (RWLF_A $=$ RWLF_B $=$ RWLT_A $=$ RWLT_B=1), while RWLs of other bitcells RPs (not shown in Figure 4) are kept low. The final voltage level on RBLF depends on stored data in bitcell A and in bitcell B (voltage level of BLTI_A and BLTI_B); if at least one of two bitcells stores ' 1 ', RBLF will be discharged to ' 0 ', if both stores a ' 0 ' (BLTI=GND, BLFI=VDD), only RBLF will be kept at VDD. This operation reproduces the truth table of a $\mathrm{N}$-input NOR logic gate. The final voltage level of RBLT is dependent on the complement of the stored data in A and B (voltage level of BLFI). If one of two bitcells stores a '0' (BLTI=GND, BLFI=VDD), RBLT is discharged, which reproduces the truth table of a N-input AND logic gate. Therefore, OR and NAND truth tables can be also reproduced by inverting RBLF and RBLT signals.

It is worth to say that while this example shows two selected bitcells, up to $\mathrm{M}$ cells ( $\mathrm{M}$ is the column length which is equal to the number of rows in the memory array) can be selected in the same column to perform operations with $\mathrm{M}$ operands, since the Boolean algebra is associative.

Figure 5 presents the proposed periphery circuitry of memory slice. A cascaded NAND gates architecture together with the MUX control signal ADDEN allow us enabling different logic and arithmetic operations. In detail, ADDEN is set to ' 1 ', only when an addition operation (ADD) has to be performed. The

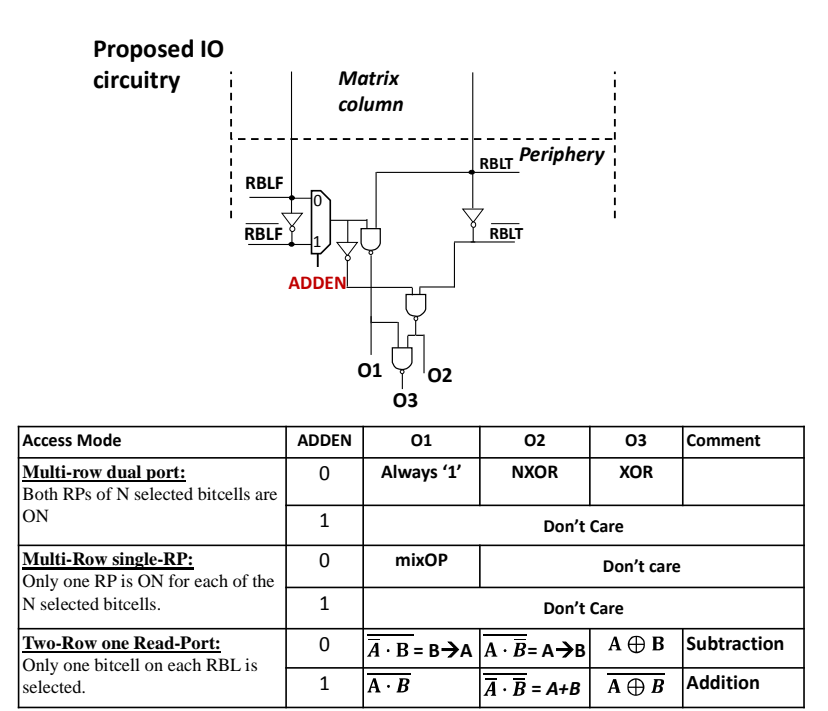

Figure 5 Periphery control and resulting outputs under different access modes.

details of arithmetic operations, ADD and subtraction (SUB), are presented later in this section.

\section{B. XOR/NXOR, COMP, Material Implication (IMP), Less than (LT) and Greater Than (GT)}

Bitcells in of a given memory slice can be accessed in 3 different ways depending on which operation(s) is (are) targeted. Multiple row selection with dual access to each bitcell allows reading all selected bitcells on both RPs as already depicted in Figure 4.b. In this access mode, if signal $\mathrm{ADDEN}=0$, periphery outputs O1, O2, O3 are equal to always ' 1 ', NXOR and XOR, respectively. If more than two bitcells in the same slice are selected, XOR output yields to a comparison operation (COMP); $\mathrm{O} 3$ is ' 1 ' if at least one among all selected bitcells stores a different data, and NXOR output O2 leads to the opposite test giving ' 1 ' if all selected bitcells store same data.

The second access mode is as follows: Multiple bitcells in a given memory slice are selected but each one is accessed through only one RP. Supposing that bitcells selected on RPF are $F_{0}, F_{1} \ldots F_{i}$ and bitcells selected through RPT are $T_{0}, T_{1} \ldots T_{k}$, a NOR is performed between $F_{0}, F_{1} \ldots F_{i}$, an AND is performed between $T_{0}, T_{1} \ldots T_{k}$. The output $\mathrm{O} 1$ is in this case equal to:

$$
\begin{array}{r}
\overline{\overline{F_{0}+F_{1} \ldots+F_{l}} \cdot T_{0} \cdot T_{1} \ldots T_{k}}=F_{0}+F_{1}+. . F_{k}+\overline{T_{0} \cdot T_{1} \ldots T_{k}} \\
=F_{0}+F_{1} \ldots F_{k}+\bar{T}_{0}+\bar{T}_{0}+\cdots \bar{T}_{k}
\end{array}
$$

This operation is named as "mixed operation" (denoted as mixOP in Figure 5). Moreover, if one bitcell is selected on each $\mathrm{RP}$, for example $F_{0}$ and $T_{1}$, output $\mathrm{O} 1$ gives $\bar{T}_{1}+F_{0}$, which is equal to material implication (IMP) denoted as $T_{1} \rightarrow F_{0}$.

Furthermore, a Boolean expression $\bar{A}+B$ is the equivalent of Less Than (LT) operator $(\mathrm{A}<\mathrm{B})$. Therefore, LT and Greater Then (GT) operations can be also performed word-wise in the proposed memory circuit with some additional gates in the array periphery; they are not detailed in this paper due to the space limitation. 


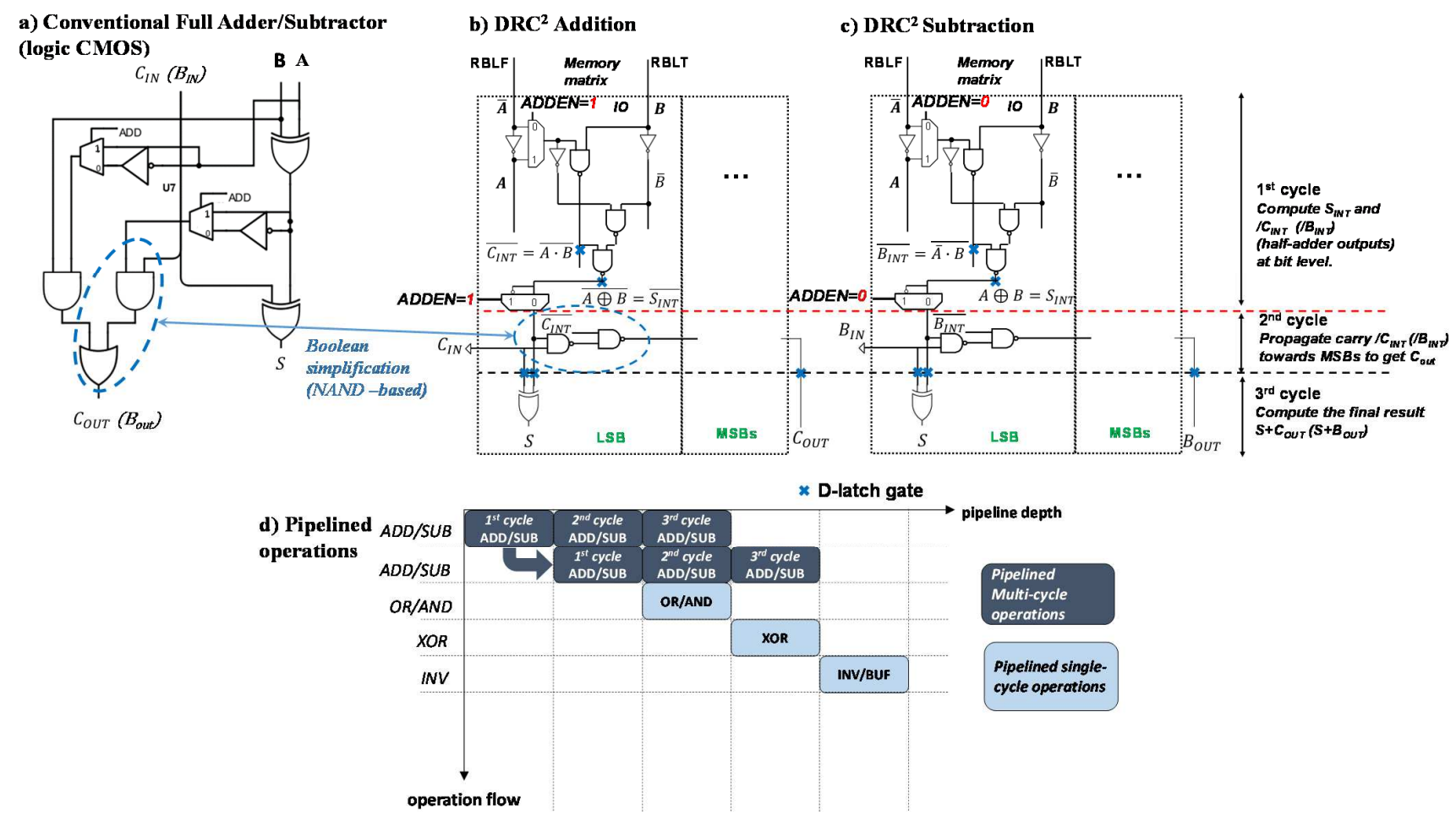

Figure 6 a) Conventional CMOS logic Full-adder/subtractor, full-adder/subtractor based on ripple-carry adder/subtractor architecture implemented in DRC 2 slice periphery $\mathrm{IO}$ b) for addition and c) for subtraction. A single ADD/SUB of 2 words of $\mathrm{K}$ bits last for 3 clock cycles. When signal ADD=1, circuit performs ADD operations, else it performs SUB operations. d) Pipelined operations in DRC ${ }^{2}$, different cycles of multi-cycle ADD/SUB operations can be pipelined together with single-cycle operations like OR, AND, XOR.

\section{Addition / Subtraction}

The last access mode denoted as two-rows-single RP, is used for arithmetic operations in the proposed circuitry. A Ripple-carry adder/subtractor, which is based on a full-adder/subtractor is implemented in the memory slice periphery allowing us to perform addition and subtraction between two K-bits word that is previously stored in the memory. A schematic of conventional a full adder/subtractor is shown Figure 6.a, which is composed of XOR, AND and OR logic gates.

Assuming bitcell $A$ is selected through RPF thus final RBLF value is equal to $\bar{A}$, and bitcell $\mathrm{B}$ is selected through RPT thus the final RBLT value is equal to $B$. If ADDEN equals to ' 1 ', a half-adder, which consists of an AND gate to compute carry (C) and a XOR gate to compute summary $(\mathrm{S})$ of the addition between operands $\mathrm{A}$ and $\mathrm{B}$, is realized in one clock cycle. Setting ADDEN to '0' allows reproducing a half-subtractor; this time the carry output becomes the borrow output (B), which is equal to $\bar{A}$. $B$. We modified the full-adder equation in order to replace the series of AND and OR gates by two NAND gates, as depicted in Figure 6.b in the circled area. This has the following benefits: Overall number of transistor decreases minimizing periphery area in the same time increasing speed, since number of stages to cross are reduced from 4 to 2 . Therefore, the carry (borrow) of the current slice, $\mathrm{C}(\mathrm{B})$, has to be read as $\bar{C}(\bar{B}$,$) ,$ which corresponds to a NAND between operands (instead of the AND as in a full adder). The following part describes implementation of a full-adder; the same is valid for fullsubtractor by replacing C (carry) with B (borrow).
A full adder can be therefore reproduced by propagating carry to columns storing LSBs of tow K-bit words. Figure $6 . \mathrm{b}$ presents how a ripple-carry adder is reproduced in memory slice allowing addition of two K-bit words. A single ADD can take 3 clock cycles for high-frequencies $(>\mathrm{GHz})$. In the first cycle, half-additions are executed; the output carry $\mathrm{C}_{\text {out_j }}$ and sum $\mathrm{S}_{\mathrm{j}}$ of memory slice $j$ are latched. In the second cycle, $\mathrm{C}_{\text {out_j } j \text { in }}$ propagated to the next slice (from LSB to MSB) allowing updating $\mathrm{C}_{\text {out } \_+1}$, and the updated $\mathrm{C}_{\text {out_j+1 }}$ is latched. This carry propagation is illustrated in Figure $6 . b$ as the input carry $C_{i n}$. Figure 6.c presents how a ripple-carry subtractor is reproduced in memory slice and borrows are propagated as the same way as a ripple-carry adder but setting ADDEN to ' 0 '.

Figure 6.d presents our pipelined ADD/SUB approach in which successive ADD/SUB area added in pipeline depth. One new operation can be added in the pipeline at every new clock cycle without any latency thanks to the 2R1W 10T SRAM bitcell.

Finally, increment (INC) and decrement (DEC) operations can also be performed, since they correspond to ADD 1 and SUB 1, respectively.

\section{Shift Operation}

Shift operation can be implemented in the proposed memory circuit by adding latches in the array periphery in order to implement a conventional CMOS shifter circuit. This part is not detailed in this paper. 


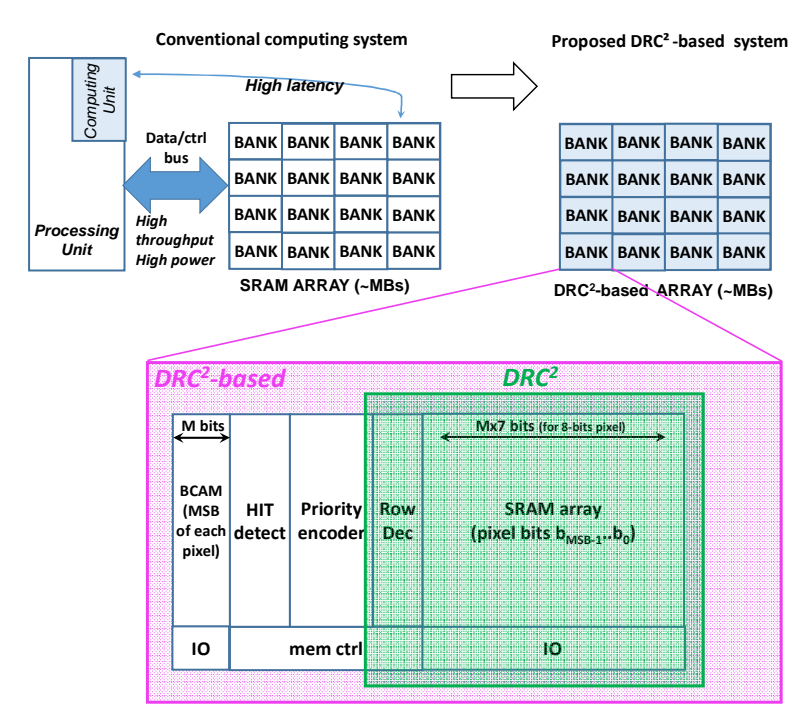

Figure 7 Conventional system based on a processing unit and SRAMs on the top left, and the proposed computing system circuit using DRC architecture coupled with a BCAM.

\section{RESULTS: IMAGE PROCESSING APPLICATION}

In modern robotic applications, the environment is modeled using occupancy grids [20]. Such grids encode occupation information using signed binary words (e.g. 8-bit words) [21]. The occupation data is then manipulated by the application as a simple mono-channel image buffer. To perform obstacle detection and velocity estimation, Bayesian occupancy filters are used. Such filters allow to estimate and predict occupancy over time. To do so, prior state (image buffer at time t-1) is mitigated then fused with current state (image buffer at time $t$ ). The mitigation can typically be implemented by looking at the sign of the data. If it is positive (bit 0 ) the data as to be decremented (by 1 or another constant value). If it is negative (bit 1), the data has to be incremented (by the same value).

Figure 7 presents on the top left a conventional system using a processing unit and a dedicated SRAM array for pixel storage, in which we can assume that the saturating increment is performed as following: Pixel values which are previously converted into signed 8-bits words are stored in a memory array. The processing unit reads sequentially each pixel and check the sign bit. Depending on the sign bit, pixel value is incremented or decremented and the new value is written back to the memory. In order to store the entire image, relatively large SRAM arrays are required which results in latencies while reading from and writing to the memory depending on the addressed memory bank location. In this work, we assume 1 latency cycle for both read and write as an average latency which may be seen as an optimistic approach. Assuming a 1RW 6T SRAM bitcell, as usually used in many microcontrollers, 1 clock cycle for read, 1 clock cycle for sign test, 1 clock cycle for increment/decrement, 1clock cycle for write back and 2 clock cycles due to latencies are required, in total leading to an overall of 6 clock cycles for one pixel. Figure 8.a presents pipelined operation flow using 1RW 6T SRAM bitcell, in which read and write operations cannot be performed in the same clock cycle. 6 clock cycles are needed to process one pixel, and each new pixel would add 3 more cycles at the end of the pipeline depth. The overall number of cycles depends on the number of pixels in the picture, $\mathrm{Np}$, and is equal to $3 * \mathrm{~Np}+3$.

Figure 7 also presents the proposed system based on $\mathrm{DRC}^{2}$ architecture coupled with a Binary Content Addressable Memory (BCAM) [15]. BCAM circuits are hardware-based search engines allowing to perform search of the memory
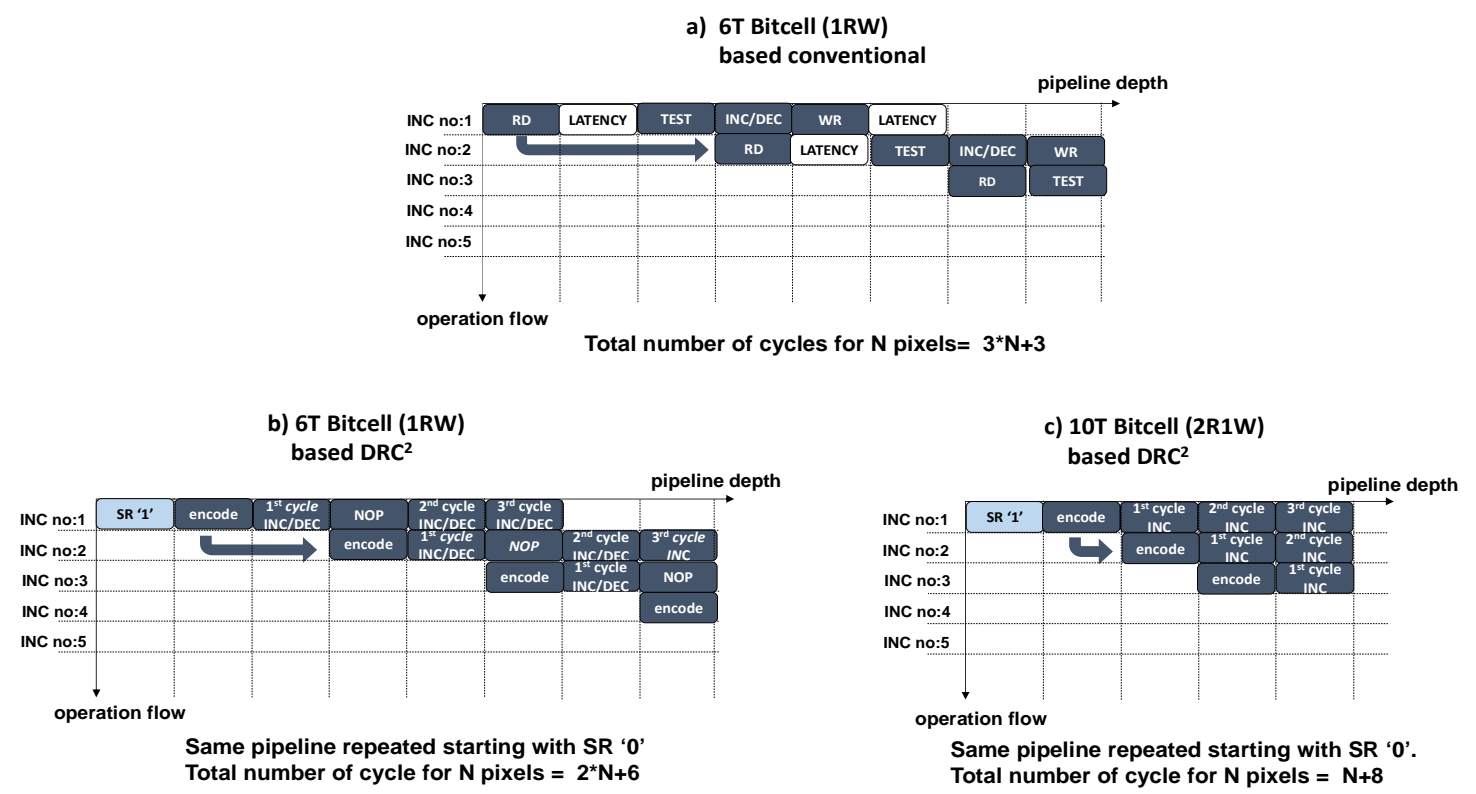

Figure 8 Pipeline stages for performing saturating increment in a pixel array using different mentioned systems. a) Conventional system using 1RW 6T SRAM, b) DRC ${ }^{2}$-based system using 1RW 6T SRAM bitcell, c) DRC ${ }^{2}$-based system using 2R1W 10T SRAM bitcell. 


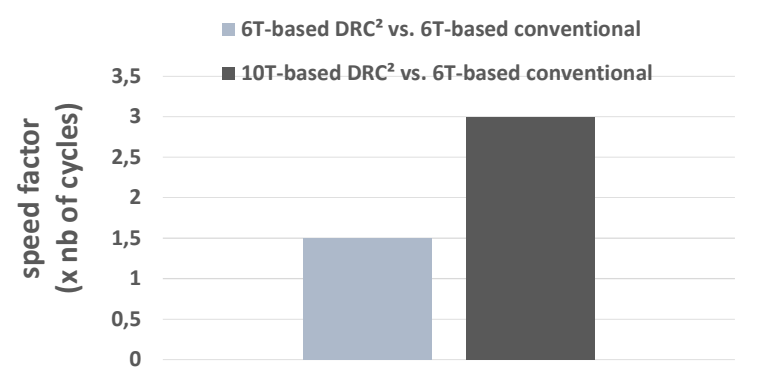

Figure 9 Clock cycle reduction factor between a conventional (using 6T SRAM bitcell) and the proposed DRC ${ }^{2}$-based system (using 6T and 10T SRAM bitcells).

content, and to match specific data in a single clock cycle. In the proposed system, MSB of each pixel is stored in the BCAM array and the rest of bits of each pixel is stored in the SRAM array. Positive signed pixels are detected by searching ' 1 ' in BCAM, while negative signed bits are detected by searching ' 0 '. The hit detection bloc outputs lines of BCAM array storing the searched sign bit, i.e. having a search hit. A priority encoder together with the memory controller would allow to sequentially read hits and increment/decrement these lines using the $\mathrm{DRC}^{2}$ increment/decrement function. In other words, given signed pixels are identified in one cycle whatever the image resolution. Priority encoder encodes one hit address and transfers the encoded address to memory controller in one cycle. The encoded hit line is discharged to ' 0 '. Memory controller sends address of the pixel that has to be incremented/decremented by 1 to the SRAM array. The same is repeated for the opposite signed bits.

As mentioned before, the SRAM array in $\mathrm{DRC}^{2}$ can be designed with any SRAM bitcell that offers differential read at the expense of overall time depending on the desired operation. Figure 8.b presents the pipeline stages for a $\mathrm{DRC}^{2}$-based system using 1RW 6T SRAM bitcell, in which one search cycle (SR) is performed at the beginning and match line encoding (encode) is performed before each 4-cycles increment/decrement operation. Since computing takes place in-memory, there is no data transmission from the memory array to an external computing unit removing all latency cycles. The $\mathrm{DRC}^{2}$-based system using 6T SRAM bitcell does not allow performing simultaneous WR and RD operations, and therefore a nooperation (NOP) cycle has to be inserted between $1^{\text {st }}$ and $2^{\text {nd }}$ cycles of an INC/DEC, which leads to an overall number of $2 * \mathrm{~Np}+3$ cycles..

As shown in section III.C, increment function takes 3 cycles using 2R1W 10T SRAM bitcell and it can be pipelined, as described in Figure 6.d. Figure 8.c presents pipeline stages of saturating increment in the $\mathrm{DRC}^{2}$-based system using $2 \mathrm{R} 1 \mathrm{~W}$ 10T SRAM bitcell, in which one search cycle is performed at the beginning and the match line encoding is performed before each 3-cycles increment operation. The total number of clock cycle is $\mathrm{Np}+8$ (versus $2 * \mathrm{~Np}+3)$.
Figure 9 presents the comparison in terms of speed between the conventional system and the $\mathrm{DRC}^{2}$-based system for a given picture resolution. In order to have a fair comparison, $\mathrm{DRC}^{2}-$ based system is considered with both bitcell-based memory arrays. It is shown that, $\approx \mathrm{x} 1.5$ speed factor is reached with DRC $^{2}$-based system using slow-but-small 1RW 6T SRAM bitcell. On the other hand, if 2R1W 10T SRAM bitcell is used in $\mathrm{DRC}^{2}$-based system, $\approx \mathrm{x} 2$ speed factor is reached w.r.t the conventional system (since 2R1W 10T SRAM bitcell allows simultaneous WR and RD operations reducing overall number of cycles in the pipeline depth).

In overall, $\mathrm{DRC}^{2}$-based systems help reducing total number of clock cycles in saturating increment/decrement function since latencies due to read from and write to memory banks are canceled by moving computing inside the memory macro. Moreover, although we are not able to quantify the gain at this stage, the suppression of the intensive data transfer between different memory banks and the computing unit, therefore suppression of all flip-flops, buffers and wires, lead to a significant power consumption reduction. In other words, the in-memory computing architecture enabled by the $\mathrm{DRC}^{2}$-based system can offer improvements in power consumption and speed, while replacing the conventional computing architecture by a configurable memory based computing circuit.

\section{DisCuSSIONS AND PERSPECTIVES}

The Von Neumann programming model, where data and instruction memory accesses are interlaced, has been very successful and a lot of technologies and tools for code generation was build using this model. More specifically, compiler are based on instruction scheduling, memory model (variable in memory, in register, in cache, etc.), data layout, etc. New parallel models such as pipelined instructions, instruction level parallelism (ILP), data parallelism (SPMD) on GPU or asynchronous multicore was already challenging as a programmer point of view and has been a very active research domain. But specific languages such as CUDA for GPU, tools such as OpenMP or OpenACC or specific libraries have tried to help programmers in this difficult task to extract different parallelism level. As said in the introduction, $\mathrm{DRC}^{2}$ will change dramatically the traffic between memory and processing core and the programming model. To the best of our knowledge, there is no specific tools or language able to generate a so high level of parallelism. We can only try to imagine different tasks on how to use this new computing in memory paradigm.

We could revisit the work already done on the bit parallel machines from the 90. The Connection Machines [18] and the MasPar was successful machines in term of parallelism usage. It supported *LISP parallel language [19] and the CMFortran which was specific languages. It was in the 90's and even if the available parallelism was important, the programming model was restrictive and difficult to exploit. Maybe work could be done in this direction. 
Another analogy come from the "SQL view" notion in databases. The SQL view allow to "see and exploit" the database through a view, which is a transformation from the initial database without modifying the initial database. The transformation can be table join or more complex transformations. We could imagine to use $\mathrm{DRC}^{2}$ in a similar way: the physical memory could be duplicated on a large memory map; each duplication corresponding to a "view". A simple computing core with a large memory system (such as the 128 bits memory from the RISC-V http://riscv.org/) could use a single view to differentiate the operations (XOR, NAND, NOR, ADD). Thus, the program become a set of DMA accesses leading to a complex treatment on big data sets.

We could imagine to add special instruction in a RISC V processor to implement these "special DMA" instruction working on large memory ranges. Of course this kind of memory should not implement a full instruction set but the compute core could also implement control operation as usual. Many applications can benefit from such a memory engine. Immediate idea are:

- Cryptography which are easily implemented as bit operations: For example, systems that are memory intensive such as "OneTimePad", could be easily implemented with a XOR between two memory locations. The DRC ${ }^{2}$ could implement those algorithms with a great energy efficiency.

- NOSQL Data bases which need a high level of parallelism: These databases need to scan datasets and select matching results. We could imagine a global selection mechanism using binary masks applied on large memory page.

- Image filtering implemented at bit level: is also a good candidate. Section IV shown an initial example of an algorithm that take advantage of the $\mathrm{DRC}^{2}$, we could imagine that many pixel level transformation implemented in graphic card (Shadows under windows, mouse cursor masking, etc) could be easily implemented.

Work are under progress to give more realistic examples of algorithms taking advantage of this $\mathrm{DRC}^{2}$.

Finally, demand for reconfigurable circuits is on rise, since modern applications should be able to adapt to different operating environments that may require loading new circuits on board. $\mathrm{DRC}^{2}$ offer high flexibility for reconfigurable computing, since logic and arithmetic operations are executed where the data is stored but not using hard-coded gates. A potential application can be in cellular networking, in which circuits should adapt to different network standards such as 3G, $4 \mathrm{G}$ and $5 \mathrm{G}$.

\section{CONCLUSION}

In this paper, we have presented a novel concept of computing circuit called $\mathrm{DRC}^{2}$, exploiting the architecture of SRAM macro. This circuit is able to perform logic and arithmetic operations with very limited data transfer to other circuits enabling a veritable in-memory computing. It should allow overcoming the well-known memory wall bottleneck and reducing data transfer-related excessive power consumption.
As a result, it is well-suited for data-intensive applications. An imaging processing test case based on saturating increment function is presented using both a conventional Von Neumann computing architecture and a DRC ${ }^{2}$-based system. The results show a significant speed gain (up to $\mathrm{x} 2$ ) by using the novel inmemory computing architecture. Furthermore, we expect a drastic power consumption reduction. DRC ${ }^{2}$ architecture paves the way for novel computing algorithms that will benefit at maximum from its features, not only for power consumption but also for high reconfigurability and increased speed.

\section{REFERENCES}

[1] Wm. A. Wulf, S. A. McKee, "Hitting the memory wall: implications of the obvious", ACM SIGARCH Comp. Arch. News, Vol 23, (1), pp 2024, 1995.

[2] M.V. Wilkes, "The memory wall and the CMOS end-point", ACM SIGARCH Comp. Arch. News, Vol 23, (4), pp.4-6, 1995.

[3] S-F. Hsiao et al., "Design of Low-Leakage Multi-Port SRAM for Register File in Graphics Processing Unit”, ISCAS, pp 2181-2184, 2014.

[4] G. Burda et al., "A 45nm CMOS 13-Port 64-Word 41b Fully Associative Content-Addressable Register File, ISSCC, pp. 286-287, 2010.

[5] M. Horowitz, "Computing's Energy Problem (and what we can do about it)”, ISSCC, pp 10-14, 2014.

[6] W. Dally, "Power Programmability, and Granularity", keynote presentation in IPDPS, 2011.

[7] HiPEAC Vision 2015 - www.hipeac.net

[8] M. Gokhale et al., "Procesing in memory: the Terasys massively parallel PIM array", IEEE Computer, vol 28 (4), pp. 23-31, 1995.

[9] Zhu et al., "A 3D-Stacked Logic-in-Memory Accelerator for ApplicationSpecific Data Intensive Computing”, 3DIC, pp 1-7, 2013.

[10] P. Jain et al., "Intelligent SRAM (ISRAM) for Improved Embedded System Performance", in proceedings of DAC, 2003.

[11] S. Kvatinsky at al., "Memristor-Based Material Implication (IMPLY) Logic: Design Principles and Methodologies “, TVLSI, vol 22 (10), October, 2014.

[12] S. Hamdioui et al., "Memristor based computation-in-memory architecture for data-intesive applications", DATE, pp 1718-1725, 2015.

[13] P. Dlugosch et al., "An Efficient and Scalable Semiconductor Architecture for Parallel Automata Processing”, IEEE TPDS, Vol. 99, pp. 3088- 3098, 2014.

[14] Mai et al., "Video-Active RAM: A Processor-in-Memory Architecture for Video Coding Applications", ISCA, pp. 161-171, 2010.

[15] Jeloka et al., "A $28 \mathrm{~nm}$ Configurable Memory (TCAM/BCAM/SRAM) Using Push-Rule 6T Bit Cell Enabling Logic-in-Memory”, JSSC,Vol 51 (99), pp 1009-121, 2016.

[16] Shibata et al., ,"A $0.5 \mathrm{~V} 25 \mathrm{MHz} 1 \mathrm{~mW} 256 \mathrm{~kb}$ MTCMOS/SOI SRAM for solar-power-operated portable personal digital equipment-sure write operation by using step-down negatively overdriven bit-line scheme", JSSC, Vol. 41 no.3, pp.728-742, 2006.

[17] Mukhopadhyay et al., "Modeling of failure probability and statistical design of SRAM array for yield enhancement in nanoscaled CMOS", IEEE TCAD, Vol. 24, no. 12, pp.1859-18880, 2005.

[18] W. D. Hillis, “The Connectino Machine”, published by MIT Press, 1989.

[19] Guy L. Steele. Jr. , al., "Connection Machine Lisp: Fine-grained Parallel Symbolic Processing", Proceedings of the 1986 ACM Conf. on LISP and Functional Programming, pp 279-277.

[20] A. Elfes, "Occupancy grids: a stochastic representation for active robot perception", Sixth Conference on Uncertainity in AI, 1990.

[21] T. Rakotovao et al., "Multi-Sensor Fusion of Occupancy Grids based on Integer Arithmetic", IEEE ICRA, 2016.

[22] C. Coué, et al. "Bayesian Occupancy Filtering for Multitarget Tracking: an automative application", Intl. Journal of Robotics Research, SAGE Publications, Vol 25 (1), pp. 19-30, 2006. 